\title{
MAXIMAL SETS OF ORTHOGONAL MEASURES ARE NOT ANALYTIC
}

\author{
DAVID PREISS AND JAN RATAJ
}

ABSTRACT. By proving the theorem given in the title we answer a question posed by D. Mauldin at the conference on Measure Theory held in Oberwolfach in 1981.

In this note we study maximal sets of pairwise orthogonal probability measures on $\langle 0,1\rangle$. The existence of such sets is guaranteed by the Kuratowski-Zorn principle. Our theorem says that such sets cannot be Borel in the weak topology, or even analytic. This answers a problem posed by D. Mauldin at the Conference on Measure Theory held in Oberwolfach in 1981 (see [2]). To study maximal sets of pairwise orthogonal measures is useful in the light of general properties of orthogonal kernels; see [2] for a survey of these results and further references.

First let us introduce some notation. Assume that $K$ is a compact metric space. $\mathscr{M}(K)$ denotes the locally convex space of all finite Borel signed measures on $K$ with weak topology. $\mathscr{M}^{+}(K)$ denotes its subspace of all positive measures and $\mathscr{P}(K)$ its subspace of all probability measures. It is well known that $\mathscr{P}(K)$ is a compact metrizable space.

If $x \in K$ we denote by $\varepsilon_{x}$ the Dirac probability measure concentrated at $x$. Let $\mathscr{D}(K)$ denote the set of all finite convex combinations of Dirac probabilities on $K$. $\mathscr{D}(K)$ is a dense subset of $\mathscr{P}(K)$.

We will also use the norm

$$
\|\mu\|=\sup \{|\mu(A)|: A \text { is a Borel subset of } K\} \quad \text { for } \mu \in \mathscr{M}(K) .
$$

The function $\mu \mapsto\|\mu\|$ is lower semicontinuous on $\mathscr{M}(K)$. Consequently, the function $(\mu, \nu) \mapsto\|\mu-\nu\|$ is lower semicontinuous on $\mathscr{P}(K) \times \mathscr{P}(K)$.

If $L$ is a compact subspace of $K$ and $B \subseteq \mathscr{P}(K)$, we define the set of restrictions $B \uparrow L=\{\mu \uparrow L: \mu \in B\}$.

Now consider the special case $K=\langle 0,1\rangle$. Assume that $r \in \mathbf{N}, I_{1}, \ldots, I_{r}$ are disjoint compact intervals in $\langle 0,1\rangle$; by an interval we always mean a nondegenerate interval, further, that $\lambda_{1}, \ldots, \lambda_{r}$ are positive numbers such that $\sum_{i=1}^{r} \lambda_{i}=1$ and

Received by the editors June 17, 1983 and, in revised form, August 22, 1983. 1980 Mathematics Subject Classification. Primary 28A33: Secondary 60B05. 
$\Delta>0$. Then we put

$$
\begin{aligned}
& U\left(r ; I_{1}, \ldots, I_{r} ; \lambda_{1}, \ldots, \lambda_{r} ; \Delta\right) \\
& \quad=\left\{\mu \in \mathscr{P}(\langle 0,1\rangle): \mu\left(\text { int } I_{i}\right)>\lambda_{i}-\Delta, \mu\left(I_{i}\right) \leqslant \lambda_{i}+\Delta, i=1, \ldots, r\right\} .
\end{aligned}
$$

If $\mu \in \mathscr{D}(\langle 0,1\rangle), \mu=\sum_{i=1}^{r} \lambda_{i} \varepsilon_{x_{i}}$, then the system of all sets

$$
U\left(r ; I_{1}, \ldots, I_{r} ; \lambda_{1}, \ldots, \lambda_{r} ; \Delta\right)
$$

such that $x_{i} \in$ int $I_{i}, i=1, \ldots, r$, and $\Delta>0$ forms a basis of open neighbourhoods of $\mu$. Consequently, every open set in $\mathscr{P}(\langle 0,1\rangle)$ contains an open set of the type (1).

For the proof we need the Banach-Mazur game as an instrument for proving residuality (see [3]). Assume that $X$ is a complete metric space and $M \subseteq X$. The Banach-Mazur game is played by two players, (A) and (B). In the first step, (A) puts $U_{1}=X$ and (B) chooses an open set $U_{1}^{\prime} \subseteq U_{1}$. In the $n$th step (A) chooses an open set $U_{n} \subseteq U_{n-1}^{\prime}$ and (B) chooses an open $U_{n}^{\prime} \subseteq U_{n}$. This defines a nonincreasing sequence of open sets. If their intersection contains an element of $M$, player (A) wins, in the opposite case (B) wins. It is well known that (A) has a winning strategy in this game if and only if $M$ is residual in $X$; the proof in [3] easily extends for the general case.

Our main result is given in the following

THEOREM. No maximal set of pairwise orthogonal Borel probability measures on the interval $\langle 0,1\rangle$ is analytic.

For the proof we will use two auxiliary lemmas:

LEMMA 1. Assume that $K \subseteq\langle 0,1\rangle$ is a finite union of compact disjoint intervals, $V \subseteq \mathscr{P}(\langle 0,1\rangle)$ an open set, and $\varepsilon \in(0,1)$. Then the set $Y=\{\zeta \in \mathscr{P}(K): \varepsilon \cdot \zeta \in V \uparrow$ $K\}$ is open in $\mathscr{P}(K)$.

Proof. Let $\zeta_{0}$ be an arbitrary measure in $Y$ and let $\mu_{0} \in V$ be an extension of $\varepsilon \cdot \zeta_{0}$. Clearly, if $\zeta \in \mathscr{P}(K)$ is sufficiently close to $\zeta_{0}$ then the measure $\mu$, defined by $\mu \uparrow K=\varepsilon \cdot \zeta, \mu \uparrow\langle 0,1\rangle \backslash K=\mu_{0} \uparrow\langle 0,1\rangle \backslash K$, extends $\varepsilon \cdot \zeta$ and is arbitrarily close to $\mu_{0}$.

LEMMA 2. Assume that $K$ is the compact set from Lemma $1, V \subseteq \mathscr{P}(\langle 0,1\rangle)$ an open set, $T \subseteq V$ is dense and open in $V, U \subseteq \mathscr{P}(K)$ is an open set, $\varepsilon \in(0,1)$, and $\Delta>0$. Assume further that for every $\zeta \in U$ the measure $\varepsilon \cdot \zeta$ can be extended into $V$ (or, equivalently $\varepsilon \cdot \zeta \in V \uparrow K)$. Then the set $Y=\{\zeta \in U$ : there exists $t \in(\varepsilon-\Delta, \varepsilon+\Delta)$, such that $t \cdot \zeta \in T \uparrow K\}$, is open and dense in $U$.

Proof. From Lemma 1 we see that $Y$ is open. We prove that it is also dense in $U$. Let $U_{0}$ be any nonempty open subset of $U$. There is $\zeta_{0} \in U_{0}$ such that (i) $\zeta_{0}$ (bdry $K$ ) $=0$. By the assumption, $\varepsilon \cdot \zeta_{0}$ can be extended at some $\mu_{0} \in V$. Let $\mu_{n}$ be any sequence of elements of $T$ converging to $\mu_{0}$. By (i), $\mu_{n} \uparrow K \rightarrow \mu_{0} \uparrow K=\varepsilon \cdot \zeta_{0}$. If $\zeta_{n}=\left(\mu_{n}(K)\right)^{-1} \cdot \mu_{n} \uparrow K$, then $\zeta_{n} \rightarrow \zeta_{0}$. Since $\mu_{n}(K) \rightarrow \varepsilon, \zeta_{n} \in U_{0} \cap Y$ for all sufficiently large $n$. 
The next lemma is the main step of the proof of our theorem.

Lemma 3. Assume that $K \subseteq\langle 0,1\rangle$ is the compact set from Lemma $1,0 \subseteq \mathscr{P}(K)$ is a nonempty open set in $\mathscr{P}(K), B \subseteq \mathscr{P}(\langle 0,1\rangle)$ is residual in an open nonempty set $G \subseteq$ $\mathscr{P}(\langle 0,1\rangle)$ and $\varepsilon \in(0,1)$. Assume further that

(1) there exists $t_{0}>\varepsilon$ such that, for every $\zeta \in 0, t_{0} \cdot \zeta \in G \uparrow K$.

Then the set $X=\{\zeta \in 0$ : there is $t>\varepsilon$ such that $t \cdot \zeta \in B \uparrow K\}$ is residual in 0 .

Proof. There are dense, open subsets $H_{n}$ in $G$ such that $\bigcap_{n=1}^{\infty} H_{n} \subseteq B$.

Let us fix a number $0<\delta<\left|t_{0}-\varepsilon\right|$. We prove the residuality of the set $X$ in 0 by describing a winning strategy in the Banach-Mazur game. We use the same notation as in the beginning of this text. Put $U_{1}=0$. Player (B) chooses open sets $U_{i}{ }^{\prime} \subseteq 0$ such that

(i) $U_{i}^{\prime} \subseteq U_{i}, i=1,2, \ldots$, and (A) chooses open sets $U_{i} \subseteq U_{i-1}^{\prime}, i=2,3, \ldots$ We will even require that

(ii) $\bar{U}_{i} \subseteq U_{i-1}, i=2,3, \ldots$ We will describe the moves of (A), no matter how (B) played in the preceding steps. In addition, we will construct open sets $V_{i} \subseteq$ $\mathscr{P}(\langle 0,1\rangle), i=1,2, \ldots$, and numbers $\varepsilon_{i}>0, i=1,2, \ldots$, with the following properties:

(iii) $\bar{V}_{i} \subseteq V_{i-1} \cap H_{i}, i=2,3, \ldots$,

(iv) $\left|\varepsilon_{i}-\varepsilon_{i-1}\right|<\delta \cdot 2^{-i}, i=2,3, \ldots$,

(v) if $\zeta \in U_{i}$, then $\varepsilon_{i} \cdot \zeta \in V_{i} \uparrow K, i=1,2, \ldots$,

(vi) $\operatorname{diam} V_{i}<i^{-1}, i=2,3, \ldots$,

(vii) if $\mu \in V_{i}$ then $\mu$ (bdry $\left.K\right)<i^{-1}, i=2,3, \ldots$.

Construction. We put $V_{1}=G$ and $\varepsilon_{1}=t_{0}$. Then, by the assumption (1) of this lemma, the objects $U_{1}, V_{1}, \varepsilon_{1}$ satisfy the condition (v). The remaining conditions have no relation to the case $i=1$.

Assume that open sets $U_{1}, U_{1}^{\prime}, V_{1}, \ldots, U_{n-1}, U_{n-1}^{\prime}, V_{n-1}$ and positive numbers $\varepsilon_{1}, \ldots, \varepsilon_{n-1}$ have already been defined and that the requirements (i)-(vii) hold. By Lemma 2 the set $X_{n}=\left\{\zeta \in U_{n-1}^{\prime}\right.$ : there is $s \in\left(\varepsilon_{n-1}-\delta \cdot 2^{-n}, \varepsilon_{n-1}+\delta \cdot 2^{-n}\right)$ such that $\left.s \cdot \zeta \in\left(V_{n-1} \cap H_{n}\right) \uparrow K\right\}$, is dense and open in $U_{n-1}^{\prime}$. Take $\zeta_{n} \in X_{n}$ such that $\zeta_{n}($ bdry $K)=0$ and take $\varepsilon_{n} \in\left(\varepsilon_{n-1}-\delta \cdot 2^{-n}, \varepsilon_{n-1}+\delta \cdot 2^{-n}\right)$ such that $\varepsilon_{n} \cdot \zeta_{n}$ can be extended to some $\mu_{n} \in V_{n-1} \cap H_{n}$; requirement (iv) holds for $\varepsilon_{n}$. There exists an open set $V_{n} \subseteq \mathscr{P}(\langle 0,1\rangle)$ such that $\mu_{n} \in V_{n}$ and the requirements (iii), (vi), (vii) hold. By Lemma 1 , if $U_{n}$ is a sufficiently small neighbourhood of $\zeta_{n}$, the requirements (ii), (v) also hold.

Finally we verify that our strategy is really winning. Let $\zeta$ be a measure from the intersection $\bigcap_{n=1}^{\infty} U_{n}$; we show that $\zeta \in X$. By (iv), the sequence $\varepsilon_{n}$ converges to some $\bar{\varepsilon}>\varepsilon$. By $(\mathrm{v}), \varepsilon_{n} \cdot \zeta$ can be extended to some $\mu_{n} \in V_{n}, n=1,2, \ldots$, and, by (iii) and (vi), there exists a measure $\mu \in \cap V_{n} \subseteq \cap H_{n} \subseteq B$ such that $\mu_{n} \rightarrow \mu$. By (vii), $\mu($ bdry $K)=0$, hence $\mu_{n} \uparrow K \rightarrow \mu \uparrow K$. On the other hand, the sequence $\mu_{n} \uparrow K=\varepsilon_{n}$ $\cdot \zeta$ converges to $\bar{\varepsilon} \cdot \zeta$; consequently, $\mu \uparrow K=\bar{\varepsilon} \cdot \zeta$. In other words, $\mu \in B$ is an extension of $\bar{\varepsilon} \cdot \zeta$. 
We now see that this strategy of the Banach-Mazur game with the set $X \subseteq 0$ is winning for $(\mathrm{A})$; hence, by the Theorem mentioned in the beginning of this paper, $X$ is residual in 0 .

Lemma 4. Assume that $G \subseteq \mathscr{P}(\langle 0,1\rangle)$ is a nonempty open convex set. Let $G_{1} \subseteq G$ and $G_{2} \subseteq G$ be open nonempty sets such that $G \subseteq \bar{G}_{1} \cup \bar{G}_{2}$, and let $\tau \in(0,1)$. Then

(a) there exists measures $\mu_{0} \in \bar{G}_{1} \cap G$ and $\nu_{0} \in G_{2}$ such that $\tau \cdot \mu_{0} \leqslant \nu_{0}$, and

(b) there exists a compact $K \subseteq(\langle 0,1\rangle)$ which is a finite union of compact disjoint intervals and there exists a nonempty open subset $0 \subseteq \mathscr{P}(K)$ such that, for every $\zeta \in 0$, $\tau \cdot \zeta \in G_{1} \uparrow K \cap G_{2} \uparrow K$.

Proof. (a) Let $\mu \in G_{1}$ and $\nu \in G_{2}$ be arbitrary measures. Put (i)

$$
\xi_{k}=\tau^{k} \cdot \mu+\left(1-\tau^{k}\right) \cdot \nu, \quad k=0,1,2, \ldots
$$

Clearly $\xi_{k} \in G$ for every $k$, because $\xi_{k}$ are convex combinations of $\mu$ and $\nu$. Let $k_{0} \geqslant 1$ be the least natural number such that $\xi_{k_{0}} \in G_{2}$; the existence of such a number follows from the fact that $\xi_{k} \rightarrow \nu \in G_{2}$. We denote $\mu_{0}=\xi_{k_{0}-1}$ (clearly $\left.\mu_{0} \in \bar{G}_{1} \cap G\right)$ and $\nu_{0}=\xi_{k_{0}}$. From (i) we get

$$
\nu_{0}=\tau^{k_{0}} \cdot \mu+\left(1-\tau^{k_{0}}\right) \cdot \nu=\tau \cdot \mu_{0}+(1-\tau) \cdot \nu,
$$

hence $\tau \cdot \mu_{0} \leqslant \nu_{0}$.

(b) We use the measures $\mu_{0}, \nu_{0}$ from (a). There exists a sequence $\mu_{n}, n=1,2, \ldots$, in $G_{1} \cap \mathscr{D}(\langle 0,1\rangle)$ such that $\mu_{n} \rightarrow \mu_{0}$. Define $\nu_{n}=\tau \cdot \mu_{n}+\left(\nu_{0}-\tau \cdot \mu_{0}\right), \quad n=$ $1,2, \ldots$ We see that $\nu_{n} \rightarrow \nu_{0}$, hence there exists $n_{0}$ such that $\nu_{n_{0}} \in G_{2}$. Let $\mu_{n_{0}}$ have the form $\mu_{n_{0}}=\sum_{i=1}^{r} \lambda_{i} \cdot \varepsilon_{x_{i}}$, where $r \in \mathbf{N}, x_{i} \in\langle 0,1\rangle, \lambda_{i}>0, i=1, \ldots, r, x_{i} \neq x_{j}$ for $i \neq j$ and $\sum_{i=1}^{r} \lambda_{i}=1$. Let $\tilde{\mu}=\mu_{n_{0}}$. The measure $\nu_{0}-\tau \cdot \mu_{0}$ can be approximated by a combination of Dirac measures $\sum_{i=r+1}^{s} \xi_{i} \cdot \varepsilon_{x_{i}}$ (where $x_{i} \in\langle 0,1\rangle \backslash\left\{x_{1}, \ldots, x_{r}\right\}$, $\xi_{i}>0, i=r+1, \ldots, s, x_{i} \neq x_{j}$ for $i \neq j$ ) such that $\tilde{\nu}=\tau \cdot \mu_{0}+\sum_{i=r+1}^{s} \xi_{i} \cdot \varepsilon_{x_{1}} \in G_{2}$.

There exist compact disjoint intervals $I_{i} \subseteq\langle 0,1\rangle, x_{i} \in$ int $I_{i}, i=1, \ldots, s$, and a number $\Delta>0$ such that neighbourhoods

$$
U(\tilde{\mu})=U\left(r ; I_{1}, \ldots, I_{r} ; \lambda_{1}, \ldots, \lambda_{r} ; \Delta\right)
$$

and

$$
U(\tilde{\nu})=U\left(s ; I_{1}, \ldots, I_{s} ; \tau \cdot \lambda_{1}, \ldots, \tau \cdot \lambda_{r}, \xi_{r+1}, \ldots, \xi_{s} ; \tau \cdot \Delta\right)
$$

fulfill the inclusions $U(\tilde{\mu}) \subseteq G_{1}$ and $U(\tilde{\nu}) \subseteq G_{2}$. Take compact intervals $K_{i} \subseteq$ int $I_{i}$, $i=1, \ldots, r$. Put $K=K_{1} \cup \cdots \cup K_{r}$ and

$$
0=\left\{\zeta \in \mathscr{P}(K): \zeta\left(K_{i}\right) \in\left(\lambda_{i}-\Delta / 2, \lambda_{i}+\Delta / 2\right), i=1, \ldots, r\right\} .
$$

We now see that for every $\zeta \in 0$ the measure $\tau \cdot \zeta$ can be extended into both neighbourhoods $U(\tilde{\mu}), U(\tilde{\nu})$, and hence into both sets $G_{1}$ and $G_{2}$.

Proof of THE Theorem. Let $A$ be a maximal set of pairwise orthogonal Borel probability measures in $\mathscr{P}(\langle 0,1\rangle)$. Assume, to the contrary, that $A$ is analytic.

For every $k \in \mathbf{N}$ let $E_{k}$ denote the space of all $k$-element subsets of $A$. We equip $E_{k}$ with its usual topology (generated, e.g., by the Hausdorff metric). Clearly, each $E_{k}$ is a continuous image of an open subset of the Cartesian product $A^{k}$, hence it is analytic. 
Whenever $k \in \mathbf{N}$ and $\varepsilon \in(0,1)$, the set of all $(\mu, j) \in \mathscr{P}(\langle 0,1\rangle) \times E_{k}$ such that $\|\mu-\nu\| \leqslant \varepsilon$ for every $\nu \in j$ is closed in $\mathscr{P}(\langle 0,1\rangle) \times E_{k}$. It follows that for every analytic set $C \subseteq E_{k}$ the set $\{(\mu, j) \in \mathscr{P}(\langle 0,1\rangle) \times C ;\|\mu-\nu\|<\varepsilon$ for every $\nu \in j\}$ is analytic and hence its first projection $H_{k, \varepsilon}(C)$ is also analytic. In addition, let $\tau \in(0, \varepsilon)$. Define $F_{k, \varepsilon}^{\tau}(C)=H_{k, \varepsilon-\tau}(C)-H_{k+1, \varepsilon}\left(E_{k+1}\right)$ and $U_{k, \varepsilon}^{\tau}=F_{k, \varepsilon}^{\tau}\left(E_{k}\right)$. Then:

(1) Whenever $k \in \mathbf{N}, \varepsilon \in(0,1), \varepsilon>\tau>0$ and $C \subseteq E_{k}$ is analytic, the set $F_{k, \varepsilon}^{\tau}(C)$ is a difference of two analytic sets and hence it has the property of Baire.

(2) If $C_{1} \subseteq E_{k}$ and $C_{2} \subseteq E_{k}$ are disjoint sets, $\mu_{1} \in F_{k, \varepsilon}^{\tau}\left(C_{1}\right)$ and $\mu_{2} \in F_{k, \varepsilon}^{\tau}\left(C_{2}\right)$, then $\left\|\mu_{1}-\mu_{2}\right\| \geqslant \tau$. (Otherwise we could find $\left\{\nu_{1}, \ldots, \nu_{k}\right\} \in C_{1}$ and $\left\{\bar{\nu}_{1}, \ldots, \bar{v}_{k}\right\} \in$ $C_{2}$ such that $\left\|\mu_{1}-\nu_{i}\right\|<\varepsilon$ and $\left\|\mu_{1}-\bar{\nu}_{i}\right\| \leqslant\left\|\mu_{1}-\mu_{2}\right\|+\left\|\mu_{2}-\bar{\nu}_{i}\right\|<\varepsilon$ for every $i=1, \ldots, k$. The disjointedness of $C_{1}$ and $C_{2}$ would imply that $\mu_{1} \in H_{k+1, \varepsilon}\left(E_{\mathrm{k}+1}\right)$.)

(3) Since $A$ is a maximal set of orthogonal measures, for every $\nu \in \mathscr{P}(\langle 0,1\rangle)$ the set $\{\mu \in A ;\|\nu-\mu\|<1\}$ is nonempty and for every $t \in(0,1)$ the set $\{\mu \in A$; $\|\nu-\mu\|<t\}$ is finite. We find $\varepsilon \in(0,1)$ such that $\|\nu-\mu\|<\varepsilon$ for some $\mu \in A$. Let $j=\{\mu \in A ;\|\nu-\mu\|<\varepsilon\}$. Since $j$ is finite, there is $\tau \in(0, \varepsilon)$ such that $\|\mu-\nu\|<\varepsilon$ - $\tau$ for each $\mu \in j$. Thus $\nu \in U_{k, \varepsilon}^{\tau}$, where $k$ is the number of elements of $j$. Consequently,

$$
\mathscr{P}(\langle 0,1\rangle)=\bigcup_{\varepsilon \in(0,1)} \bigcup_{k=1}^{\infty} \bigcup_{\tau \in(0, \varepsilon)} U_{k, \varepsilon}^{\tau} .
$$

We now prove that $U_{k, \varepsilon}^{\tau}$ is of first category for every $k \in \mathbf{N}, \varepsilon \in(0,1), \varepsilon>\tau>0$. Assume, to the contrary, that $U_{k, \varepsilon}^{\tau}$ is of second category. Then, by the property of Baire, it is residual in some nonempty open set (see [1]). We can assume that $U_{k, \xi}^{\tau}$ is residual in a convex nonempty open set $G \subseteq \mathscr{P}(\langle 0,1\rangle)$. Denote by $Z$ the maximal open set in $E_{k}$ such that $F_{k, \varepsilon}^{\tau}(Z)$ is of first category in $G$. We see that $F=E_{k} \backslash Z$ has at least two different elements, because the set $F_{k, \varepsilon}^{\tau}(\{j\})$ is nowhere dense for every $j \in E_{k}$. Take a partition $F=F_{1} \cup F_{2}$, where $F_{1}$ and $F_{2}$ are Borel subsets of $F$ with nonempty interiors. Put $A_{1}=F_{k, \varepsilon}^{\tau}\left(F_{1}\right), A_{2}=F_{k, \varepsilon}^{\tau}\left(F_{2}\right)$. Clearly, $A_{1}$ and $A_{2}$ are both of second category in $G$; by (1) they have the property of Baire, and hence they are residual in some open sets. Denote by $G_{1}, G_{2}$ the maximal open subsets in $G$, such that $A_{1}, A_{2}$ are residual in $G_{1}, G_{2}$, respectively. Clearly $G \subseteq \bar{G}_{1} \cup \bar{G}_{2}$. By Lemma 4 there exists a compact subset $K \subseteq\langle 0,1\rangle$ which is a finite union of compact disjoint intervals, and a nonempty open set $0 \subseteq \mathscr{P}(K)$ such that for every $\zeta \in 0$ the measure $(1-\tau) \cdot \zeta$ can be extended into $G_{1}$ and $G_{2}$. By Lemma 3 , the sets

$$
X_{1}=\left\{\zeta \in 0: \text { there is } t_{1}>1-\tau \text { such that } t_{1} \cdot \zeta \in A_{1} \uparrow K\right\}
$$

and

$$
X_{2}=\left\{\zeta \in 0: \text { there is } t_{2}>1-\tau \text { such that } t_{2} \cdot \zeta \in A_{2} \uparrow K\right\}
$$

are residual in 0 . Hence their intersection is nonempty and there exist $\zeta \in 0$, $\mu_{1} \in A_{1}, \mu_{2} \in A_{2}$ and $t_{1}, t_{2}>1-\tau$ such that $\mu_{1} \uparrow K=t_{1} \cdot \zeta$ and $\mu_{2} \uparrow K=t_{2} \cdot \zeta$. Hence $\left\|\mu_{1}-\mu_{2}\right\|<\tau$. But by (2) we see that $\left\|\mu_{1}-\mu_{2}\right\| \geqslant \tau$. This contradiction proves that $U_{k, \varepsilon}^{\tau}$ is of first category.

In (3) it suffices to take only countable unions, hence the whole space $\mathscr{P}(\langle 0,1\rangle)$ is of first category. This contradiction proves our Theorem. 


\section{REFERENCES}

1. K. Kuratowski, Topology I, Academic Press, New York and London, 1968.

2. D. Mauldin, D. Preiss and H. v. Weizsäcker, A survey of problems and results concerning orthogonal transition kernels, Measure Theory (Proc. Conf., Oberwolfach, 1981), Lecture Notes in Math., Vol. 945. Springer-Verlag, New York, 1982.

3. J. C. Oxtoby, Measure and category, Springer-Verlag, New York, 1980.

Faculty of Mathematics and Physics, Charles University, Sokolovska 83, 186000 Prague 8. CZEChoslovakia 